\title{
THE SUPERPOSITION OF ALTERNATING ON-OFF FLOWS AND A FLUID MODEL
}

\author{
By ZBigniew PaLmowski ANd Tomasz Rolski ${ }^{1}$ \\ University of Wroctaw
}

\begin{abstract}
An on-off process is a $0-1$ process $\xi_{t}$ in which consecutive 0 -periods $\left\{T_{0, n}\right\}$ alternate with 1-periods $\left\{T_{1, n}\right\}(n=1,2, \ldots)$. The on and off time sequences are independent, each consisting of i.i.d. r.v.s. By the superposed flow, we mean the process $Z_{t}=\sum_{\ell=1}^{N} r^{\ell} \xi_{t}^{\ell}$, where $r^{\ell}>0$ and $\left\{\xi_{t}^{1}\right\}, \ldots,\left\{\xi_{t}^{N}\right\}$ are independent on-off flows. The process $\xi_{t}^{\ell}$ is not Markovian; however, with the age component $\eta_{t}^{\ell}$, the process $w_{t}^{\ell}=\left(\xi_{t}^{\ell}, \eta_{t}^{\ell}\right)$ is a piecewise deterministic Markov process. In this paper we study the buffer content process for which the tail of its steady-state distribution $\Psi(b)$ fulfills inequality $C_{-} e^{-\gamma b} \leq \Psi(b) \leq C_{+} e^{-\gamma b}$, where $\gamma>0$ is the solution of some basic nonlinear system of equations.
\end{abstract}

1. Introduction. Fluid models with exponential on and off times were intensively studied by many authors beginning with the pioneering papers of Anick, Mitra and Sondhi (1982) in the homogeneous case and Stern and Elwalid (1991) for a nonhomogeneous case [see also a survey by Kulkarni (1995)]. In this paper we study fluid models with general on and off times and, under some assumptions, we derive exponential lower and upper bounds for the tail of the steady-state distribution of the buffer content. Bensaou, Guibert, Roberts and Simonian (1994) and Guibert (1994) studied fluid models using the Beneš-Borovkov equation. However, their results for nonexponential on and off times are computationally quite complex. Guibert and Simonian (1995) found approximations for the tail of the steady-state distribution of the buffer content using large deviations techniques; however, the form of the rate function derived in their paper can be utilized only numerically. Other references on related topics are Palmowski and Rolski (1996), Kulkarni (1994), Asmussen and Rubinstein (1995) and Whitt (1993). Nonexponential estimates for the tail of the buffer content distribution were studied, for instance, by Heath, Resnick and Samorodnitsky (1996) and Jelenkovič and Lazar (1996).

By an on-off flow we mean a $0-1$ process $\xi_{t}$, in which consecutive 0-periods $\left\{T_{0, n}\right\}$ alternate with 1-periods $\left\{T_{1, n}\right\}(n=1,2, \ldots)$. Random variables $\left\{T_{0, n}\right\}$ are off times and $\left\{T_{1, n}\right\}$ are on times. We assume that both sequences are independent, each consisting of i.i.d. random variables. In this paper we consider $N$ flows $\xi_{t}^{1}, \ldots, \xi_{t}^{N}$. The flows are not supposed to be identically distributed and we denote the generic on and off times of the $\ell$ th flow by $T_{1}^{\ell}$

Received June 1996; revised September 1997.

${ }^{1}$ Supported by KBN Grant 1026/803/95/08.

AMS 1991 subject classifications. Primary 60K25; secondary 68M20, 90B22.

Key words and phrases. Queueing fluid model, exponential bound, on-off flow, superposition of on-off flows, generator. 
and $T_{0}^{\ell}$, respectively $(\ell=1, \ldots, N)$. If $\mathbb{E}\left(T_{0}^{\ell}+T_{1}^{\ell}\right)<\infty$, then there exists the (time) stationary on-off flow $\xi_{t}^{\ell}$.

In this paper we consider the fluid model with input rate $Z_{t}=\sum_{\ell=1}^{N} r^{\ell} \xi_{t}^{\ell}$ and constant output rate $c$, wherein the buffer content process $X_{t}$ is governed by the equation

$$
\frac{d X_{t}}{d t}= \begin{cases}Z_{t}-c, & \text { for } X_{t}>0 \\ \left(Z_{t}-c\right)_{+}, & \text {for } X_{t}=0\end{cases}
$$

It is known [see, e.g., Kulkarni and Rolski (1994)] that if the stability condition

$$
\sum_{\ell=1}^{N} p^{\ell} r^{\ell}<c
$$

holds, where

$$
p^{\ell}=\frac{\mathbb{E} T_{1}^{\ell}}{\mathbb{E} T_{0}^{\ell}+\mathbb{E} T_{1}^{\ell},}
$$

then there exists $\Psi(b)=\lim _{t \rightarrow \infty} \mathbb{P}\left(X_{t} \geq b\right)$ and

$$
\begin{aligned}
\Psi(b) & =\mathbb{P}\left(\sup _{t \leq 0} \int_{t}^{0}\left(Z_{s}-c\right) d s \geq b\right) \\
& =\mathbb{P}\left(\sup _{t \geq 0} \int_{0}^{t}\left(Z_{s}-c\right) d s \geq b\right) .
\end{aligned}
$$

In this case, $\lim _{b \rightarrow \infty} \Psi(b)=0$ and we study how fast $\Psi(b)$ converges to 0 . We also suppose that

$$
c<\sum_{\ell=1}^{N} r^{\ell} ;
$$

otherwise, the buffer is empty in the steady state. Notice that $p^{\ell}$ is the steady-state probability that the $\ell$ th flow is in the state on. The main result of this paper are two-sided exponential bounds for $\Psi(x)$. For the $\ell$ th source $(\ell=1, \ldots, N)$, let $F_{1}^{\ell}$ denote the distribution of $T_{1}^{\ell}$ and let $F_{0}^{\ell}$ denote the distribution of $T_{0}^{\ell}$; the corresponding moment generating functions are $\hat{F}_{1}^{\ell}$ and $\hat{F}_{0}^{\ell}$, respectively. We assume that the distributions are absolutely continuous with densities $f_{1}^{\ell}$ and $f_{0}^{\ell}$ and the corresponding hazard rate functions

$$
r_{1}^{\ell}(x)=\frac{f_{1}^{\ell}(x)}{1-F_{1}^{\ell}(x)}, \quad r_{0}^{\ell}(x)=\frac{f_{0}^{\ell}(x)}{1-F_{0}^{\ell}(x)},
$$

respectively. If flows are identically distributed, then the superscript $\ell$ is omitted. Such flows are called homogeneous flows, in contrast with the more general case of heterogeneous flows.

Throughout this paper we make the following assumption about the existence of the solution of a system of equations, which we call the basic system 
of nonlinear equations (BSNL). There exist $\gamma>0$ and $c^{\ell}>p^{\ell} r^{\ell}(\ell=1, \ldots, N)$ fulfilling the following system of equations:

$$
\begin{aligned}
\hat{F}_{1}^{\ell}\left(-\gamma\left(c^{\ell}-r^{\ell}\right)\right) \hat{F}_{0}^{\ell}\left(-\gamma c^{\ell}\right) & =1, \quad \ell=1, \ldots, N, \\
\sum_{\ell=1}^{N} c^{\ell} & =c .
\end{aligned}
$$

We call $\gamma$ the adjustment coefficient and we discuss this system later in Section 5. It is clear that $F_{1}^{\ell}$ cannot be heavy-tailed, and in Theorem 1.1 we impose even stronger conditions. For the homogeneous case it suffices to assume that there exists $\gamma>0$ such that

$$
\hat{F}_{1}\left(-\gamma\left(\frac{c}{N}-r\right)\right) \hat{F}_{0}\left(-\gamma \frac{c}{N}\right)=1
$$

The main result of the paper is the following theorem.

THEOREM 1.1. Suppose that

$$
\inf _{y \rightarrow \infty} \mathbb{E}\left[\exp \left(-\gamma c^{\ell}\left(T_{0}^{\ell}-y\right)\right) \mid T_{0}^{\ell}>y\right]>0
$$

and

$$
\sup _{y \rightarrow \infty} \mathbb{E}\left[\exp \left(\gamma\left(r^{\ell}-c^{\ell}\right)\left(T_{1}^{\ell}-y\right)\right) \mid T_{1}^{\ell}>y\right]<\infty
$$

for $\ell=1, \ldots, N$. Then for some constants $0<C_{-} \leq C_{+}$,

$$
C_{-} e^{-\gamma x} \leq \Psi(x) \leq C_{+} e^{-\gamma x}, \quad x \geq 0 .
$$

A sufficient condition for (1.6) is

$$
\liminf _{x \rightarrow \infty} r_{0}^{\ell}(x)=\rho_{0}^{\ell}>0
$$

and for (1.7) is

$$
\limsup _{x \rightarrow \infty} r_{1}^{\ell}(x)=\rho_{1}^{\ell}>0 \quad \text { and } \quad \rho_{1}^{\ell}>\gamma\left(r^{\ell}-c^{\ell}\right) .
$$

The proof is given in Section 3, wherein we also give forms for $C_{-}$and $C_{+}$in formulas (3.12) and (3.13), respectively. We give a comment on conditions (1.8) and (1.9) in Section 5.

For the proof of Theorem 1.1 we use a Markovian theory of on-off flows. Process $\xi_{t}$ is not Markovian alone, but it is Markovian with the supplementary age component $\eta_{t}$. Thus, the process $w_{t}=\left(\xi_{t}, \eta_{t}\right)$ is a Markov process, which is a piecewise deterministic (PD) Markov process - a class introduced by Davis $(1984,1993)$. Moreover, the (extended) generator $\mathbf{Q}$ of the Markov process $w_{t}$ is known. We recall needed concepts and results from PD processes in Section 2. In the proof we use a form of exponential martingales and a perturbation theorem from the Appendix to define a new underlying probability measure. The change of measure technique, called twisting in large deviation theory [see 
Shwartz and Weiss (1995)], is standard for such purposes [see, e.g., Palmowski and Rolski (1996) and Asmussen (1994)].

2. Process $\boldsymbol{w}_{\boldsymbol{t}}$. Formally we define the process $w_{t}=\left(\xi_{t}, \eta_{t}\right)$ as follows. Let $\tau_{n}, n=1,2, \ldots$, be the sequence of switchover epochs. It is defined regarding the initial condition $w_{0}=\left(\xi_{0}, \eta_{0}\right)$. If $\xi_{0}=0$ and $\eta_{0}=y$, then $\tau_{1}$ has distribution $F_{0}(d t+y) / \bar{F}_{0}(y)$ and is independent of $\left\{T_{0, n}\right\},\left\{T_{1, n}\right\}$. Then we define recursively

$$
\tau_{n+1}= \begin{cases}\tau_{n}+T_{0, n / 2}, & \text { if } n \text { is even } \\ \tau_{n}+T_{1,\lceil n / 2\rceil}, & \text { if } n \text { is odd }\end{cases}
$$

and

$$
\xi_{t}= \begin{cases}0, & \text { if } \tau_{n} \leq t<\tau_{n+1} \text { and } n \text { is even, } \\ 1, & \text { if } \tau_{n} \leq t<\tau_{n+1} \text { and } n \text { is odd }\end{cases}
$$

Similarly, if $\xi_{0}=1$ and $\eta_{0}=y$, then $\tau_{1}$ has distribution $F_{1}(d t+y) / \bar{F}_{1}(y)$ and it is independent of $\left\{T_{0, n}\right\}$ and $\left\{T_{1, n}\right\}$ and we define recursively

$$
\tau_{n+1}= \begin{cases}\tau_{n}+T_{1, n / 2}, & \text { if } n \text { is even, } \\ \tau_{n}+T_{0,\lceil n / 2\rceil}, & \text { if } n \text { is odd }\end{cases}
$$

and

$$
\xi_{t}= \begin{cases}1, & \text { if } \tau_{n} \leq t<\tau_{n+1} \text { and } n \text { is even } \\ 0, & \text { if } \tau_{n} \leq t<\tau_{n+1} \text { and } n \text { is odd }\end{cases}
$$

We define the supplementary age process by $\eta_{t}=t-\tau_{n}$ if $\tau_{n} \leq t<\tau_{n+1}$. For all initial conditions $i=0,1$ and $y \geq 0$, the process $w_{t}$ is Markov and we denote the underlying probability measure by $\mathbb{P}^{(i, y)}$. If $\mu(d i, d y)$ is a probability on $\{0,1\} \times \mathbb{R}_{+}$, then we denote

$$
\mathbb{P}_{\mu}=\int \mathbb{P}^{(i, y)} \mu(d i, d y) .
$$

The process $w_{t}$ is stationary if we choose

$$
\begin{aligned}
\mu(i, d y) & =\pi(i, d y) \\
& =\frac{1}{\mathbb{E}\left(T_{0}+T_{1}\right)} \bar{F}_{i}(y) d y .
\end{aligned}
$$

Generator $\mathbf{Q}$ of $w_{t}$ and its domain $\mathscr{D}(\mathbf{Q})$ are defined as follows. We denote by $C_{b}\left(\{0,1\} \times \mathbb{R}_{+}\right)$all continuous and bounded functions $g:\{0,1\} \times \mathbb{R}_{+} \rightarrow \mathbb{R}$. For $g, g^{*} \in C_{b}\left(\{0,1\} \times \mathbb{R}_{+}\right)$we denote

$$
M_{g, g^{*}}\left(w_{t}\right)=g\left(\xi_{t}, \eta_{t}\right)-g(i, y)-\int_{0}^{t} g^{*}\left(\xi_{s}, \eta_{s}\right) d s, \quad t \geq 0 .
$$

We look for all functions $g, g^{*} \in C_{b}\left(\{0,1\} \times \mathbb{R}_{+}\right)$such that $M_{g, g^{*}}\left(w_{t}\right), t \geq 0$, is a $\mathbb{P}^{(i, y)}$-martingale for all $(i, y)$, and then we denote this family of $g$ by $\mathscr{D}(\mathbf{Q})$ 
and the mapping $\mathbf{Q}: g \rightarrow g^{*}$ we call a (full) generator. The result of Theorem 26.14 from Davis (1993) adapted to the process $w_{t}$ says that for $i=0,1$,

$$
(\mathbf{Q} g)(i, x)=\frac{\partial}{\partial x} g(i, x)+r_{i}(x)(g(1-i, 0)-g(i, x))
$$

and $\mathscr{D}(\mathbf{Q})$ consists of all functions $g(i, y) \in C_{b}\left(\{0,1\} \times \mathbb{R}_{+}\right)$, such that $g(i, y)$ are absolute continuous on $\left[0, s_{i}^{*}\right)$, where $s_{i}^{*}=\inf \left\{t: F_{i}(t)=1\right\}$.

REMARK 2.1. Following Davis [(1993), Remark 26.16] we can consider unbounded functions $g$. Then we have that $\mathscr{D}(\mathbf{Q})$ consist of all functions $g(i, y)$, such that $g(i, y)$ are absolute continuous on $\left[0, s_{i}^{*}\right)$, where $s_{i}^{*}=\inf \left\{t: F_{i}(t)=\right.$ $1\}$ and $\mathbb{E} \mid g\left(i, T_{i}^{\ell} \mid<\infty(\ell=1, \ldots, N ; i=0,1)\right.$.

3. Proof of Theorem 1.1. We first prove it for $N=1$ and then we show how to adapt the proof for the general case. In this case we do not write superscript $\ell$. In the first lemma we consider the following system of differential equations:

$$
(\mathbf{Q} h)(i, y)=\beta(r i-c) h(i, y), \quad i=0,1 .
$$

LEMMA 3.1. The smallest $\beta<0$ such that there exists $h \in \mathscr{D}(\mathbf{Q})$ and $\inf _{i, y} h(i, y)>0$ fulfilling (3.1) is $\beta=-\gamma$. Then

$$
\begin{aligned}
h(1, y) & =\frac{\exp (-\gamma(r-c) y)}{\bar{F}_{1}(y)} \int_{y}^{\infty} \exp (\gamma(r-c) z) f_{1}(z) d z \\
& =\mathbb{E}\left[\exp \left(\gamma(r-c)\left(T_{1}-y\right)\right) \mid T_{1}>y\right]
\end{aligned}
$$

and

$$
\begin{aligned}
h(0, y) & =\hat{F}_{1}(-\gamma(c-r)) \frac{\exp (\gamma c y)}{\bar{F}_{0}(y)} \int_{y}^{\infty} \exp (-\gamma c z) f_{0}(z) d z \\
& =\hat{F}_{1}(-\gamma(c-r)) \mathbb{E}\left[\exp \left(-\gamma c\left(T_{0}-y\right)\right) \mid T_{0}>y\right] .
\end{aligned}
$$

PRoOF. Solving system (3.1) with initial conditions we get

$$
\begin{array}{r}
h(i, y)=\frac{\exp (\beta(i r-c) y)}{\bar{F}_{i}(y)}\left[h(i, 0)-h(1-i, 0) \int_{0}^{y} \exp (-\beta(i r-c) z) f_{i}(z) d z\right], \\
i=0,1, y \geq 0 .
\end{array}
$$

Since we want $\inf _{i, y} h(i, y)>0$, therefore, for all $i=0,1$ and $y \geq 0$,

$$
h(i, 0)-h(1-i, 0) \int_{0}^{y} \exp (-\beta(i r-c) z) f_{i}(z) d z>0,
$$

from which, passing with $y \rightarrow \infty$, we obtain

$$
h(i, 0)-h(1-i, 0) \int_{0}^{\infty} \exp (-\beta(i r-c) z) f_{i}(z) d z \geq 0 .
$$


Choosing $h(0,0)=1$ we write

$$
\begin{aligned}
1-h(1,0) \hat{F}_{0}(\beta c) & \geq 0, \\
h(1,0)-\hat{F}_{1}(\beta(c-r)) & \geq 0
\end{aligned}
$$

or

$$
\hat{F}_{1}(\beta(c-r)) \leq h(1,0) \leq \hat{F}_{0}^{-1}(\beta c) .
$$

Therefore, we look for the smallest negative $\beta$ for which

$$
\hat{F}_{1}(\beta(c-r)) \leq \hat{F}_{0}^{-1}(\beta c)
$$

or $H(\beta) \leq 1$, where

$$
H(x)=\hat{F}_{1}(x(c-r)) \hat{F}_{0}(x c) .
$$

The function $H$ is strictly $\log$ convex because $\log H$ is a sum of two strictly convex functions $\log \hat{F}_{i}(i=0,1)$ [see Kingman (1961)]. Moreover, it is equal to 1 at $x=0$ and, in view of assumption (1.1), its derivative at zero is positive. Therefore, the function is strictly less than 1 in the interval $(-\gamma, 0)$ and greater than 1 in $(-\infty,-\gamma)$. Thus, $h(1,0)=\hat{F}_{1}(-\gamma(c-r))=\hat{F}_{0}^{-1}(-\gamma c)$ and hence

$$
\begin{aligned}
& h(1, y)=\frac{\exp (-\gamma(r-c) y)}{\bar{F}_{1}(y)} \int_{y}^{\infty} \exp (\gamma(r-c) z) f_{1}(z) d z, \\
& h(0, y)=\hat{F}_{1}(-\gamma(c-r)) \frac{\exp (\gamma c y)}{\bar{F}_{0}(y)} \int_{y}^{\infty} \exp (-\gamma c z) f_{0}(z) d z .
\end{aligned}
$$

We now show that $h \in \mathscr{D}(\mathbf{Q})$ and $\inf _{i, y} h(i, y)>0$. Clearly, functions $h(i, y)$ $(i=1,2)$ are absolute continuous and so it suffices to show that they are bounded away from 0 and $\infty$. Thus,

$$
\begin{aligned}
& h(1, y)=\int_{y}^{\infty} \frac{\exp (\gamma(r-c)(z-y))}{\bar{F}_{1}(y)} f_{1}(z) d z \geq \int_{y}^{\infty} \frac{f_{1}(z)}{\bar{F}_{1}(y)} d z=1, \\
& h(0, y) \leq \hat{F}_{1}(-\gamma(c-r)) .
\end{aligned}
$$

Moreover, $h(1, y)$ is bounded above by $(1.7)$ and $h(0, y)$ is cutoff zero by (1.6).

Using (3.1) we have

$$
(\mathbf{Q} h)\left(\xi_{s}, \eta_{s}\right)=-\gamma\left(r \xi_{s}-c\right) h\left(\xi_{s}, \eta_{s}\right) .
$$

From Proposition 3.2 of Ethier and Kurtz (1986) (recalled in the Appendix in Proposition A.1), we have that

$$
M_{t}=\frac{h\left(w_{t}\right)}{h\left(w_{0}\right)} \exp \left(-\int_{0}^{t} \frac{\mathbf{Q} h\left(w_{s}\right)}{h\left(w_{s}\right)}\right) d s=\frac{h\left(w_{t}\right)}{h\left(w_{0}\right)} \exp \left(\gamma \int_{0}^{t}\left(Z_{s}-c\right) d s\right)
$$

is a martingale. Define now a family of probability measures on $\left(\Omega, \mathscr{F}_{t}^{w}\right)$ by $d \tilde{\mathbb{P}}_{t}^{w_{0}}=M_{t} d \mathbb{P}_{t}^{w_{0}}$, where $\mathbb{P}_{t}^{w_{0}}=\mathbb{P}_{\mid \mathscr{T}_{t}^{w}}^{w_{0}}(t \geq 0)$. Since $\left\{M_{t}, t \geq 0\right\}$ is a multiplica- 
tive functional of the process $w_{t}$ from Kunita (1976), we get that this family defines exactly one probability measure $\tilde{\mathbb{P}}$ on $\left(\Omega, \bigvee_{t \geq 0} \mathscr{F}_{t}^{w}\right)$, where $\bigvee_{t \geq 0} \mathscr{F}_{t}^{w}$ is the smallest $\sigma$-field generated by all elements of $\mathscr{F}_{t}(t \geq 0)$ and $w_{t}$ is also a Markov process. By $\widetilde{\mathbb{E}}$ we denote the expectation corresponding to $\widetilde{\mathbb{P}}$.

LEMMA 3.2. The process $w_{t}$ on $\left(\Omega, \bigvee_{t>0} \mathscr{F}_{t}^{w}, \tilde{\mathbb{P}}\right)$ is an alternating on-off flow with on-time and off-time distributions $\tilde{\tilde{F}}_{1}$ and $\tilde{F}_{0}$, respectively, with densities

$$
\tilde{f}_{0}(x)=\frac{e^{-\gamma c x}}{\hat{F}_{0}(-\gamma c)} f_{0}(x), \quad \tilde{f}_{1}(x)=\frac{e^{-\gamma(c-r) x}}{\hat{F}_{0}(-\gamma(c-r))} f_{1}(x)
$$

and

$$
r \frac{\tilde{\mathbb{E}} T_{1}}{\tilde{\mathbb{E}}\left(T_{1}+T_{0}\right)}-c>0 .
$$

PRoOF. From the perturbation theorem in the Appendix,

$$
\begin{aligned}
(\tilde{\mathbf{Q}} g)(i, y)= & \frac{\partial g(i, y)}{\partial y}+\frac{g(i, y)}{h(i, y)} \frac{\partial h(i, y)}{\partial y}+\gamma(r i-c) g(i, y) \\
& +r_{i}(y)\left[\frac{h(1-i, 0)}{h(i, y)} g(1-i, 0)-g(i, y)\right] \\
= & \frac{\partial g(i, y)}{\partial y}+\tilde{r}_{i}(y)[g(1-i, 0)-g(i, y)],
\end{aligned}
$$

where

$$
\begin{aligned}
& \tilde{r}_{0}(y)=\frac{\exp (-\gamma c y) f_{0}(y)}{\int_{y}^{\infty} \exp (-\gamma c z) f_{0}(z) d z}, \\
& \tilde{r}_{1}(y)=\frac{\exp (-\gamma(c-r) y) f_{1}(y)}{\int_{y}^{\infty} \exp (-\gamma(c-r) z) f_{1}(z) d z} .
\end{aligned}
$$

From (2.4) we get (3.6). We now compute that

$$
\begin{aligned}
& \tilde{\mathbb{E}} T_{1}=\frac{\int_{0}^{\infty} z \exp (-\gamma(c-r) z) f_{1}(z) d z}{\hat{F}_{1}(-\gamma(c-r))}=\frac{\hat{F}_{1}^{\prime}(-\gamma(c-r))}{\hat{F}_{1}(-\gamma(c-r))}, \\
& \tilde{\mathbb{E}} T_{0}=\frac{\int_{0}^{\infty} z \exp (-\gamma c z) f_{0}(z) d z}{\hat{F}_{0}(-\gamma c)}=\frac{\hat{F}_{0}^{\prime}(-\gamma c)}{\hat{F}_{0}(-\gamma c)} .
\end{aligned}
$$

To demonstrate that the new drift is positive, we compute

$$
\begin{aligned}
r \frac{\tilde{\mathbb{E}} T_{1}}{\tilde{\mathbb{E}} T_{1}+\tilde{\mathbb{E}} T_{0}}-c & =r \frac{\hat{F}_{1}^{\prime}(-\gamma(c-r)) \hat{F}_{0}(-\gamma c)}{\hat{F}_{1}^{\prime}(-\gamma(c-r)) \hat{F}_{0}(-\gamma c)+\hat{F}_{0}^{\prime}(-\gamma c) \hat{F}_{1}(-\gamma(r-c))}-c \\
& =-\frac{H^{\prime}(-\gamma)}{\hat{F}_{1}^{\prime}(-\gamma(c-r)) \hat{F}_{0}(-\gamma c)+\hat{F}_{0}^{\prime}(-\gamma c) \hat{F}_{1}(-\gamma(r-c))} .
\end{aligned}
$$

The derivative of function $H$ defined in (3.4) at $x=-\gamma$ is clearly negative while the denominator is positive. Therefore the above expression is positive. 
We are now ready to give the proof of Theorem 1.1 for $N=1$. Let $\tau(b)=$ $\min \left\{t \geq 0: \int_{0}^{t}\left(r \xi_{s}-c\right) d s \geq b\right\}$. Thus from $d \mathbb{P}_{t}=M_{t}^{-1} d \tilde{\mathbb{P}}_{t}$, following the idea from Asmussen (1994), using the fact that by Lemma 3.2 stopping time $\tau(b)$ is $\widetilde{\mathbb{P}}^{(i, y)}$-a.s. finite and the strong Markov property, we can write

$$
\begin{aligned}
\Psi_{(i, y)}(b) & =\mathbb{P}^{(i, y)}(\tau(b)<\infty) \\
& =\tilde{\mathbb{E}}_{(i, y)}\left[\frac{h(i, y)}{h\left(w_{\tau(b)}\right)} \exp \left(\int_{0}^{\tau(b)} \frac{\mathbf{Q} h\left(w_{s}\right)}{h\left(w_{s}\right)} d s\right) ; \tau(b)<\infty\right] \\
& =\tilde{\mathbb{E}}_{(i, y)}\left[\frac{h(i, y)}{h\left(w_{\tau(b)}\right)}\right] e^{-\gamma b} .
\end{aligned}
$$

Hence, by (2.1),

$$
\Psi(b)=e^{-\gamma b} \frac{1}{\mathbb{E}\left(T_{0}+T_{1}\right)} \sum_{i=0}^{1} \int_{0}^{\infty} \bar{F}_{i}(y) h(i, y) \tilde{\mathbb{E}}_{(i, y)}\left[\frac{1}{h\left(\xi_{\tau(b)}, \eta_{\tau(b)}\right)}\right] d y .
$$

LEMMA 3.3. For $N=1$, constants $C_{+}$and $C_{-}$from Theorem 1.1 are

$$
C_{+}=\frac{1}{\mathbb{E}\left(T_{0}+T_{1}\right)} \frac{1}{\inf _{i, y} h(i, y)} \frac{r}{c} \frac{\hat{F}_{1}(\gamma(r-c))-1}{\gamma(r-c)}
$$

and

$$
C_{-}=\frac{1}{\mathbb{E}\left(T_{0}+T_{1}\right)} \frac{1}{\sup _{i, y} h(i, y)} \frac{r}{c} \frac{\hat{F}_{1}(\gamma(r-c))-1}{\gamma(r-c)} .
$$

Proof. For deriving $C_{+}$, we write

$$
\begin{aligned}
\Psi(b)= & e^{-\gamma b} \sum_{i=0}^{1} \frac{\mathbb{E} T_{i}}{\mathbb{E}\left(T_{0}+\mathbb{E} T_{1}\right)} \int_{0}^{\infty} \frac{\bar{F}_{i}(y) d y}{\mathbb{E} T_{i}} h(i, y) \tilde{\mathbb{E}}_{(i, y)}\left[\frac{1}{h\left(\xi_{\tau(b)}, \eta_{\tau(b)}\right)}\right] \\
\leq & e^{-\gamma b} \frac{1}{\mathbb{E}\left(T_{0}+T_{1}\right)} \frac{1}{\inf _{i, y} h(i, y)} \\
& \times\left(\int_{0}^{\infty} \exp (-\gamma(r-c) y) d y \int_{y}^{\infty} \exp (\gamma(r-c) z) f_{1}(z) d z\right. \\
& \left.\quad+\hat{F}_{1}(\gamma(r-c)) \int_{0}^{\infty} \exp (\gamma c y) d y \int_{y}^{\infty} \exp (-\gamma c z) f_{0}(z) d z\right) \\
= & \exp (-\gamma b) \frac{1}{\mathbb{E}\left(T_{0}+T_{1}\right)} \frac{1}{\inf _{i, y} h(i, y)} \\
& \quad \times\left(\frac{\hat{F}_{1}(\gamma(r-c))-1}{\gamma(r-c)}+\frac{\hat{F}_{1}(\gamma(r-c))\left(1-\hat{F}_{0}(-\gamma c)\right)}{\gamma c}\right) \\
= & \exp (-\gamma b) \frac{1}{\mathbb{E}\left(T_{0}+T_{1}\right)} \frac{1}{\inf _{i, y} h(i, y)}\left(\frac{\hat{F}_{1}(\gamma(r-c))-1}{\gamma}\right)\left(\frac{1}{r-c}+\frac{1}{c}\right) .
\end{aligned}
$$

Similar derivations prove $C_{-}$. 
We now consider general $N$. Consider Markov process $W_{t}=\left(\boldsymbol{\xi}_{t}, \boldsymbol{\eta}_{t}\right)$. We make it stationary if the initial distribution is

$$
\pi(\mathbf{i}, d \mathbf{y})=\prod_{\ell=1}^{N} \pi^{\ell}\left(i^{\ell}, d y^{\ell}\right),
$$

where $\pi^{\ell}$ is defined in (2.3) for the $\ell$ th flow. Under probability measure $\mathbb{P}_{\pi}=$ $\int \mathbb{P}^{(\mathbf{i}, \mathbf{y})} d \pi$ the process $Z_{t}=\sum_{\ell=1}^{N} r^{\ell} \xi_{t}^{\ell}$ is the input rate and $Z_{t}-c$ is the net rate. Let $\tau(b)=\min \left\{t \geq 0: \int_{0}^{t}\left(Z_{s}-c\right) d s \geq 0\right\}$ and $\mathbf{h}(\mathbf{i}, \mathbf{y})=\prod_{\ell=1}^{N} h^{\ell}\left(i^{\ell}, y^{\ell}\right)$. Following (3.5), each process

$$
M_{t}^{\ell}\left(c^{\ell}\right)=\frac{h^{\ell}\left(w_{t}^{\ell}\right)}{h^{\ell}\left(w_{0}^{\ell}\right)} \exp \left(\gamma \int_{0}^{t}\left(r^{\ell} \xi_{s}^{\ell}-c^{\ell}\right) d s\right), \quad t \geq 0,
$$

is a $\left(\mathbb{P}^{\left(i^{\ell}, y^{\ell}\right)}, \mathscr{F}_{t}^{w^{\ell}}\right)$-martingale provided the $\ell$ th equation of BSNL (1.3) holds. Consider now

$$
M_{t}=\prod_{\ell=1}^{N} M_{t}^{\ell}\left(c^{\ell}\right), \quad t \geq 0
$$

Since $\sum_{\ell=1}^{N} c^{\ell}=c$,

$$
M_{t}=\frac{\mathbf{h}\left(W_{t}\right)}{\mathbf{h}(\mathbf{i}, \mathbf{y})} \exp \left(\gamma \int_{0}^{t}\left(Z_{s}-c\right) d s\right)
$$

and $M_{t}$ is a $\left(\mathbb{P}^{(\mathbf{i}, \mathbf{y})}, \mathscr{F}_{t}^{W}\right)$-martingale. Similarly as for $N=1$, we define new probability measure $\tilde{\mathbb{P}}^{(\mathbf{i}, \mathbf{y})}$ by $d \tilde{\mathbb{P}}_{t}^{(\mathbf{i}, \mathbf{y})}=M_{t} d \mathbb{P}_{t}^{(\mathbf{i}, \mathbf{y})}$ on $\left(\Omega, \mathscr{F}_{t}^{W}\right)$, where now $\mathscr{F}_{t}^{W}$ is the history of $W_{t}$ up to $t$. The extension of (3.10) for general $N$ is

$$
\begin{aligned}
\Psi(b)=e^{-\gamma b} \prod_{\ell=1}^{N} \frac{1}{\mathbb{E}\left(T_{0}^{\ell}+T_{1}^{\ell}\right)} \sum_{\mathbf{i} \in 2^{\{0,1\}}} \int_{\mathbb{R}_{+}^{N}} \prod_{\ell=1}^{N} \bar{F}_{i^{\ell}}\left(y^{\ell}\right) h^{\ell}\left(i^{\ell}, y^{\ell}\right) \\
\times \tilde{\mathbb{E}}_{(\mathbf{i}, \mathbf{y})}\left[\frac{1}{\mathbf{h}\left(\boldsymbol{\xi}_{\tau(b)}, \boldsymbol{\eta}_{\tau(b)}\right)}\right] d \mathbf{y}
\end{aligned}
$$

and hence for the upper bound we have

$$
\Psi(b) \leq \prod_{\ell=1}^{N} \frac{1}{\mathbb{E}\left(T_{0}^{\ell}+T_{1}^{\ell}\right)}\left(\sum_{i^{\ell}=0}^{1} \int_{0}^{\infty} \bar{F}_{i^{\ell}}\left(y^{\ell}\right) h^{\ell}\left(i^{\ell}, y^{\ell}\right) d y^{\ell}\right) \frac{1}{\inf _{i, y} h^{\ell}(i, y)} .
$$

The proof of the lower bound is similar. Hence we obtain

$$
\prod_{\ell=1}^{N} C_{-}^{\ell} e^{-\gamma b} \leq \Psi(b) \leq \prod_{\ell=1}^{N} C_{+}^{\ell} e^{-\gamma b}, \quad b \geq 0,
$$

where

$$
C_{+}^{\ell}=\frac{1}{\inf _{i, y} h^{\ell}(i, y)}\left(\frac{1}{\mathbb{E}\left(T_{0}^{\ell}+T_{1}^{\ell}\right)} \frac{r^{\ell}}{c^{\ell}} \frac{\hat{F}_{1}^{\ell}\left(\gamma\left(r^{\ell}-c^{\ell}\right)\right)-1}{\gamma\left(r^{\ell}-c^{\ell}\right)}\right)
$$


and

$$
C_{-}^{\ell}=\frac{1}{\sup _{i, y} h^{\ell}(i, y)}\left(\frac{1}{\mathbb{E}\left(T_{0}^{\ell}+T_{1}^{\ell}\right)} \frac{r^{\ell}}{c^{\ell}} \frac{\hat{F}_{1}^{\ell}\left(\gamma\left(r^{\ell}-c^{\ell}\right)\right)-1}{\gamma\left(r^{\ell}-c^{\ell}\right)}\right) .
$$

4. Gaussian asymptotics. In this section we find the asymptotics for $\gamma_{N}$ in the homogeneous on-off model parametrized by the number of sources $N$ with $F_{0}$ and $F_{1}$ fixed, input rate $r_{N}$ and output rate $c_{N}$. For some $c, r>0$, let

$$
r_{N}=\frac{r}{\sqrt{N}}, \quad c_{N}=c+p r \sqrt{N}
$$

Under some mild conditions [see Szczotka (1980)], the sequence of processes

$$
\tilde{Z}_{N}(t)=\frac{\sum_{i=1}^{N} \xi_{i}(t)-N p}{\sqrt{N}}
$$

considered as random elements on $D[0, \infty)$ converges in distribution to a Gaussian process $\{\tilde{Z}(t)\}$ with mean 0 and covariance function $R(t)$ of the process $\left\{\xi_{i}(t)\right\}$. It can be proved [see Kulkarni and Rolski (1994)] that in this case, under conditions (4.1),

$$
X_{N} \stackrel{\mathscr{D}}{\rightarrow} X
$$

where $X_{N}$ and $X$ are, respectively, the steady-state buffer contents in the $N$ th on-off model and in the fluid model driven by the Gaussian process $\{r \tilde{Z}(t)\}$. For the Gaussian fluid model with net rate $\{r \tilde{Z}(t)-c\}$, Dębicki and Rolski (1995) showed

$$
\mathbb{P}(X>b) \leq C e^{-\gamma b}+o(\exp (-\gamma x)),
$$

where constant $C$ is given explicitly and

$$
\gamma=\frac{c}{r^{2} \int_{0}^{\infty} R(t) d t}
$$

It can be demonstrated [see, e.g., Kopociński (1973), page 294, or Kopociński (1967)] that, for $\left\{\xi_{i}(t)\right\}$ and hence also for $\{\tilde{Z}(t)\}$,

$$
\int_{0}^{\infty} R(t) d t=\mu_{01}^{2}\left(\mu_{12}-\mu_{11}^{2}\right)+\mu_{11}^{2}\left(\mu_{02}-\mu_{01}^{2}\right)
$$

where

$$
\mu_{0 i}=\int_{0}^{\infty} x^{i} d F_{0}(x), \quad \mu_{1 i}=\int_{0}^{\infty} x^{i} d F_{1}(x) .
$$

In the following proposition we demonstrate that, from Theorem 1.1, straightforward computations lead us to exactly the same limit.

Proposition 4.1. Suppose that the third moments of $T_{0}$ and $T_{1}$ are finite. If $\gamma_{N}$ and $\gamma$ are given by (1.5) and (4.2), respectively, and (4.1) holds, then $\gamma_{N} \rightarrow \gamma$ as $N \rightarrow \infty$. 
Proof. Applying the Taylor formula to

$$
\hat{F}_{1}\left(-\gamma_{N}\left(\frac{c_{N}}{N}-r_{N}\right)\right) \hat{F}_{0}\left(-\gamma_{N} \frac{c_{N}}{N}\right)=1
$$

we get

$$
\left(1+a_{1}(N) \gamma_{N}+\frac{1}{2} a_{2}(N) \gamma_{N}^{2}+o\left(N^{-1}\right)\right)\left(1+b_{1}(N) \gamma_{N}+\frac{1}{2} b_{2}(N) \gamma_{N}^{2}+o\left(N^{-1}\right)\right)=1,
$$

where

$$
a_{i}(N)=\mu_{0 i}\left(\frac{c_{N}}{N}\right)^{i}, \quad b_{i}(N)=\mu_{1 i}\left(\frac{c_{N}}{N}-r_{N}\right)^{i},
$$

which is equivalent by (4.1) to

$$
\left(\mu_{01}+\mu_{11}\right) \gamma_{N} c-\frac{\gamma_{N}^{2} r^{2}}{2\left(\mu_{01}+\mu_{11}\right)^{2}}\left(\mu_{01}^{2} \mu_{12}+\mu_{02} \mu_{11}^{2}-2 \mu_{01}^{2} \mu_{11}^{2}\right)+o\left(N^{-1}\right)=0 .
$$

Hence by (4.3),

$$
\gamma_{N} \rightarrow \frac{c}{r^{2}}\left[\mu_{01}^{2}\left(\mu_{12}-\mu_{11}^{2}\right)+\mu_{11}^{2}\left(\mu_{02}-\mu_{01}^{2}\right)\right]^{-1}=\frac{c}{r^{2} \int_{0}^{\infty} R(t) d t}
$$

5. System of nonlinear equations. In this section we consider the BSNL in the following form: for some $c^{1}, \ldots, c^{N}>0$ such that $\sum_{\ell=1}^{N} c^{\ell}=c$ and $\gamma>0$,

$$
\hat{F}_{1}^{\ell}\left(-\gamma\left(c^{\ell}-r^{\ell}\right)\right) \hat{F}_{0}^{\ell}\left(-\gamma c^{\ell}\right)=1, \quad \ell=1, \ldots, N
$$

Since $c^{\ell}<r^{\ell}$, from Jensen inequality we have

$$
\exp \left(-\gamma\left(\left(c^{\ell}-r^{\ell}\right) \mathbb{E} T_{1}+c \mathbb{E} T_{0}\right)\right)<\mathbb{E} \exp \left(-\gamma\left(\left(c^{\ell}-r^{\ell}\right) T_{1}+c^{\ell} T_{0}\right)\right)=1 .
$$

Hence $-\gamma\left(\left(c^{\ell}-r^{\ell}\right) \mathbb{E} T_{1}+c^{\ell} \mathbb{E} T_{0}\right)<0$, which yields $c^{\ell}>p^{\ell} r^{\ell}$, explaining the assumption for $c^{\ell}$ made in Section 1 .

Consider first the homogeneous case with the single equation

$$
H(-\gamma)=\hat{F}_{1}(-\gamma(c-r)) \hat{F}_{0}(-\gamma c)=1 .
$$

This equation appeared in Asmussen and Rubinstein (1995) in the context of optimal change of measure. Clearly, if $\lim _{s \rightarrow s^{*}} \hat{F}_{1}(s)=\infty$ for some $0 \leq s^{*} \leq \infty$, then (5.2) has the unique solution, because in this case, for continuous, convex function $H$, we have $\lim _{x \rightarrow x_{0}} H(x)=\infty$ for some $-\infty \leq x_{0} \leq 0$. We point out that this is fulfilled for the large class of phase-type distributions [for the definition of phase-type distributions, we refer to Neuts (1981)]. Indeed, let $T_{1}$ be phase type with representation $(\boldsymbol{\alpha}, \mathbf{T})$, where $\boldsymbol{\alpha}$ is a probability vector and $\mathbf{T}$ is a transient intensity matrix. Then

$$
\begin{aligned}
& \bar{F}_{1}(x)=\boldsymbol{\alpha} \exp (x \mathbf{T}) \mathbf{e}, \\
& \hat{F}_{1}(s)=-\boldsymbol{\alpha}(s \mathbf{I}+\mathbf{T})^{-1} \mathbf{t}^{\circ}, \\
& f_{1}(x)=\boldsymbol{\alpha} \exp (x \mathbf{T}) \mathbf{t}^{\circ},
\end{aligned}
$$


where $\mathbf{e}$ is a column vector consisting of $1 \mathrm{~s}$ and $\mathbf{t}^{\circ}=-\mathbf{T e}$. We assume that $\mathbf{T}$ is a subintensity matrix (off-diagonal entries are nonnegative and at least one row sums up to a strictly negative number) and hence the Perron-Frobenius eigenvalue $\lambda_{1}$ is negative and for all remaining eigenvalues $\Re\left(\lambda_{j}\right)<\Re\left(\lambda_{1}\right)$ $(j=2, \ldots, n)$. Hence from (5.4) we get that $\lim _{s \rightarrow-\lambda_{1}} \hat{F}_{1}(s)=\infty$. Moreover, for phase-type distributions, conditions (1.8) and (1.9) are also fulfilled. In fact, let $F_{1}$ be phase type. Then from (1.3) and (5.4) we get

$$
\gamma(c-r)>\lambda_{1} \text {. }
$$

Suppose that $\phi_{i}$ and $\xi_{i}$ are, respectively, the left and right eigenvectors corresponding to $\lambda_{i}$. Moreover, if the eigenvectors $\left\{\boldsymbol{\phi}_{1}, \ldots, \boldsymbol{\phi}_{n}\right\}$ are independent (e.g., in the case when eigenvalues of $\mathbf{T}$ are distinct), then

$$
\exp (x \mathbf{T})=\sum_{i=1}^{n} e^{\lambda_{i} x} \boldsymbol{\xi}_{i} \boldsymbol{\phi}_{i}
$$

Using this representation, inequalities (5.3), (5.5) and definition of vector $\mathbf{t}^{\circ}$,

$$
\lim _{x \rightarrow \infty} r_{1}(x)=\frac{\boldsymbol{\alpha} \xi_{1} \phi_{0} \mathbf{t}^{\circ}}{\boldsymbol{\alpha} \xi_{1} \phi_{0} \mathbf{e}}=-\lambda_{1} .
$$

Hence condition (1.9) follows from (5.6). Similarly, we can show that for phasetype distribution $F_{0}$, condition (1.8) is fulfilled. Thus, all assumptions of Theorem 1.1 are fulfilled if on and off times are phase type.

Sufficient conditions for the unique existence of the general BSNL are given in the following proposition:

Proposition 5.1. Let $\lim _{s \rightarrow s_{\ell}^{*}} \hat{F}_{1}^{\ell}(s)=\infty$ for some $0 \leq s_{\ell}^{*} \leq \infty(\ell=1, \ldots, N)$. Then there exist the unique $\gamma>0, c^{1}>p^{1} r^{1}, \ldots, c^{N}>p^{N} r^{N}$ solving BSNL (1.3).

PRoOF. We first recall that $H^{\ell}\left(x, c^{\ell}\right)=\hat{F}_{1}^{\ell}\left(x\left(c^{\ell}-r^{\ell}\right)\right) \hat{F}_{0}\left(x c^{\ell}\right)(\ell=1, \ldots, N)$ are continuous and strictly convex functions and define function $\kappa^{\ell}\left(c^{\ell}\right)$ by the equation $H^{\ell}\left(-\kappa^{\ell}\left(c^{\ell}\right), c^{\ell}\right)=1$. Each function $H^{\ell}\left(x, c^{\ell}\right)$ is decreasing with respect to $c^{\ell}$. Hence $\kappa^{\ell}\left(c^{\ell}\right)$ is a continuous increasing function. Moreover, the following argument shows that $\kappa^{\ell}\left(c^{\ell}\right) \rightarrow 0$ as $c^{\ell} \searrow p^{\ell} r^{\ell}$. If $c^{\ell} \searrow p^{\ell} r^{\ell}$, then $\left.(d / d x) H^{\ell}\left(x, c^{\ell}\right)\right|_{x=0} \rightarrow 0$. Supposing that for some $\kappa_{0}^{\ell}>0$ there is $\kappa^{\ell}\left(c^{\ell}\right) \rightarrow \kappa_{0}^{\ell}$ as $c^{\ell} \searrow p^{\ell} r^{\ell}$, we would have $H^{\ell}\left(x, p^{\ell} r^{\ell}\right)=1$ for $-\kappa_{0}^{\ell}<x \leq 0$, which is impossible, because each $H^{\ell}\left(x, c^{\ell}\right)$ is strictly convex. Therefore, the graph of function $H^{\ell}$ moves at level 1 from 0 to certain constant as $c^{\ell}$ move from $p^{\ell} r^{\ell}$ to $c$. Thus, there exist constants $c^{1}>p^{1} r^{1}, \ldots, c^{N}>p^{N} r^{N}$ and $\sum_{\ell=1}^{N} c^{\ell}=c$ such that these graphs have one common point $-\gamma$ at level 1 , which is the solution of BSNL.

Similarly, as for the one source case, we get the following corollary:

COROLLARY 5.1. If $F_{0}^{\ell}$ and $F_{1}^{\ell}(\ell=1, \ldots, N)$ are phase-type distributions, then there exists the unique solution of BSNL. Moreover, conditions (1.8) and (1.9) hold. 
However, there are cases when the system has no solutions as the following example shows. The simple case is when $F_{0}$ and $F_{1}$ are degenerated at $\mathbb{E} T_{0}$ and $\mathbb{E} T_{1}$, respectively. Then $\exp \left(-\gamma\left((c-r) \mathbb{E} T_{1}+c \mathbb{E} T_{0}\right)\right)=1$ yields $-\gamma\left((c-r) \mathbb{E} T_{1}+c \mathbb{E} T_{0}\right)=0$, which is possible only if $c=r p$. However, this is impossible because $c>r p$. The explanation of this fact is that for the periodic on-off stream the buffer content process is clearly bounded and, therefore, exponential bounds are too strong. The above example can be extended easily to nondegenerated random variables taking for the $F_{1}$ and $F_{0}$ uniform distribution over $\left(\mathbb{E} T_{i}-\varepsilon, \mathbb{E} T_{i}+\varepsilon\right)(i=0,1)$ for sufficiently small $\varepsilon>0$. Then (5.2) reads

$$
H_{\varepsilon}(-\gamma)=\exp \left(-\gamma\left((c-r) \mathbb{E} T_{1}+c \mathbb{E} T_{0}\right)\right)=\frac{\sinh (-\gamma \epsilon(c-r))}{-\gamma \epsilon(c-r)} \frac{\sinh (-\gamma \epsilon c)}{-\gamma \epsilon c}=1 .
$$

However, the left-hand side is strictly less than 1 for all positive $\gamma>0$ (see Figure 1).

Consider now the case when there exist groups of size $N_{1}, \ldots, N_{m}$. Within groups, the input rate is $r_{i}>0$, and $F_{1, i}$ and $F_{0, i}$ are on and off time distributions, respectively; namely,

$$
\begin{gathered}
r^{1}=\cdots=r^{N_{1}}=r_{1}, \\
r^{N_{1}+1}=\cdots=r^{N_{1}+N_{2}}=r_{2}, \\
\vdots \\
r^{N_{1}+\cdots+N_{m-1}+1}=\cdots=r^{N_{1}+\cdots+N_{m}}=r_{m}
\end{gathered}
$$

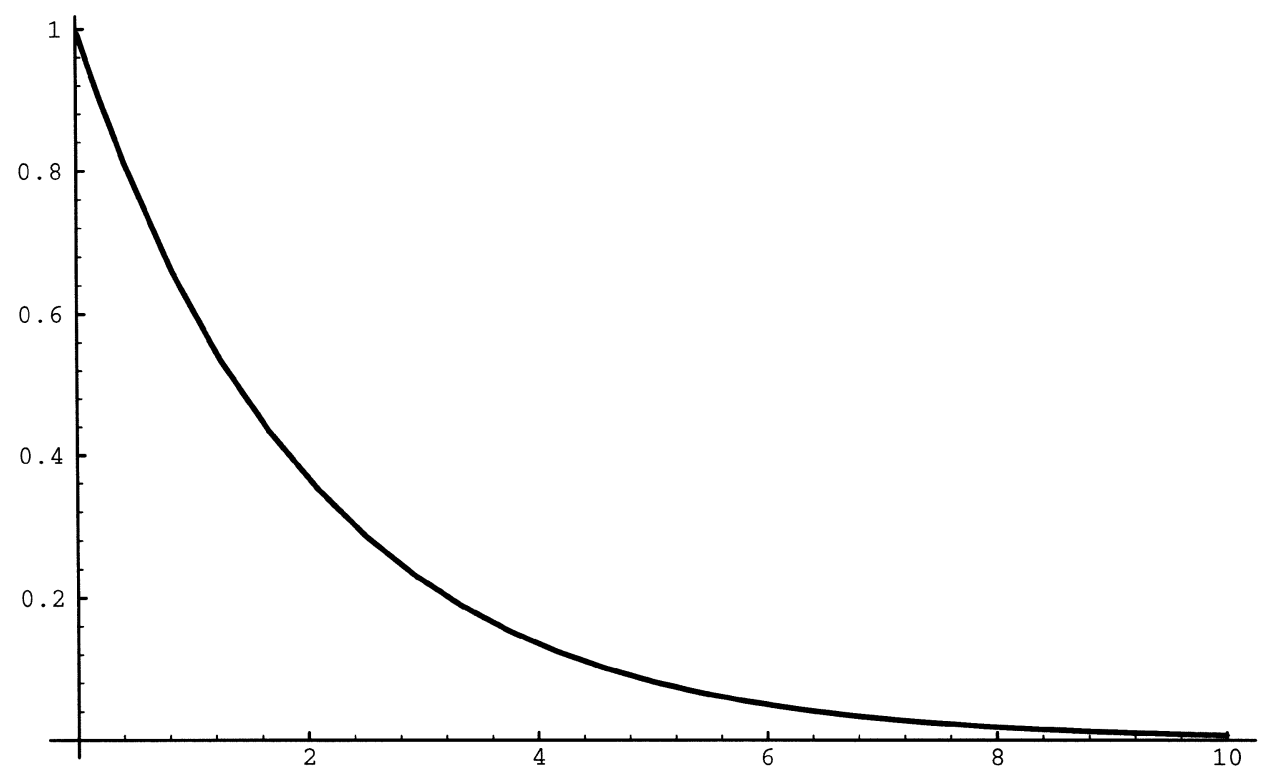

FIG. 1. 
and for $i=0,1$

$$
\begin{gathered}
F_{i}^{1}=\cdots=F_{i}^{N_{1}}=F_{i, 1}, \\
F_{i}^{N_{1}+1}=\cdots=F_{i}^{N_{1}+N_{2}}=F_{i, 2}, \\
\vdots \\
F_{i}^{N_{1}+\cdots+N_{m-1}+1}=\cdots=F_{i}^{N_{1}+\cdots+N_{m}}=F_{i, m} .
\end{gathered}
$$

Then the BSNL is reduced to the following system: there exists for some $c_{1}, \ldots, c_{m}>0$ such that $\sum_{j=1}^{m} N_{j} c_{j}=c$ and $\gamma>0$,

$$
\hat{F}_{1, j}\left(-\gamma\left(\frac{c_{j}}{N_{j}}-r_{j}\right)\right) \hat{F}_{0, j}\left(-\gamma \frac{c_{j}}{N_{j}}\right)=1, \quad j=1, \ldots, m .
$$

To compare different fluid models, we made some numerical experiments. To simplify calculations we consider only the homogeneous case. Following Anick, Mitra and Sondhi (1982), we take $r_{N}=1, T_{0} \sim \operatorname{Exp}(0.4)$ and $c_{N}=16.666$ for $N=30,50, c_{N}=33.333$ for $N=85,100, c_{N}=66.666$ for $N=150,200$ (see Table 1). In the first case, $T_{1}$ has exponential distribution $T_{1} \sim \operatorname{Exp}(1)$; in the second case, $T_{1}$ has Erlang distribution $\operatorname{Erl}(2,2)$; in the third case, $T_{1}$ has hyperexponential distribution $p \operatorname{Exp}(2)+(1-p) \operatorname{Exp}((2-2 p) /(2-p))$, where $p=0.1$. In all cases, $E T_{1}=1$. It is easy to verify that in the model considered by Anick, Mitra and Sondhi (both $T_{0}$ and $T_{1}$ have exponential distribution), (1.5) has the same solution as was given by Anick, Mitra and Sondhi (1982) or recently by Palmowski and Rolski (1996).

We can justify the order $\gamma_{\text {Hyper }}<\gamma_{\operatorname{Exp}}<\gamma_{\operatorname{Erl}}$ as follows. Let $F_{1}^{*} \sim \operatorname{Erl}(2,2)$, $F_{1} \sim \operatorname{Exp}(1)$ and $F_{0} \sim \operatorname{Exp}(0.4)$. Since $\operatorname{Erl}(2,2)$ has increasing failure rate [see Szekli (1995), page 17], so $\operatorname{Erl}(2,2) \prec_{i c x} \operatorname{Exp}(1)$. Thus,

$$
\hat{F}_{1}^{*}\left(-\gamma_{\operatorname{Exp}}\left(\frac{c}{N}-r\right)\right) \hat{F}_{0}\left(-\gamma_{\operatorname{Exp}} \frac{c}{N}\right) \leq \hat{F}_{1}\left(-\gamma_{\operatorname{Exp}}\left(\frac{c}{N}-r\right)\right) \hat{F}_{0}\left(-\gamma_{\operatorname{Exp}} \frac{c}{N}\right)=1
$$

\begin{tabular}{|c|c|c|c|c|c|c|}
\hline \multirow[b]{2}{*}{$T_{1}$} & \multicolumn{2}{|c|}{$c_{N}=16.666$} & \multicolumn{2}{|c|}{$c_{N}=33.333$} & \multicolumn{2}{|c|}{$c_{N}=66.666$} \\
\hline & $\boldsymbol{N}=\mathbf{3 0}$ & $N=\mathbf{5 0}$ & $N=85$ & $N=100$ & $N=150$ & $N=\mathbf{2 0 0}$ \\
\hline $\operatorname{Exp}$ & 1.5299 & 0.2999 & 0.6251 & 0.3 & 0.9 & 0.3 \\
\hline Erl & 2.304 & 0.41 & 0.8786 & 0.41 & 1.294 & 0.41 \\
\hline Hyperexp & 1.4677 & 0.2909 & 0.6043 & 0.291 & 0.8677 & 0.291 \\
\hline
\end{tabular}

and hence $\gamma_{\mathrm{Erl}} \geq \gamma_{\mathrm{Exp}}$. A similar argument, together with Theorem E from Szekli [(1994), page 17], proves the first inequality.

TABLE 1

Numerical comparisons of $\gamma$ 


\section{APPENDIX}

We now recall a theorem from the theory of Markov processes. The result was used by Ethier and Kurtz (1993) and Fukushima and Stroock (1986). For the proof, we refer also to Palmowski (1996). Let $\left\{X_{t}, t \geq 0\right\}$ be an $E$-valued Markov process on $\left(\Omega, \mathscr{F},\left\{\mathscr{F}_{t}\right\}_{t \geq 0},\left\{\mathbb{P}_{x}\right\}_{x \in \mathbb{R}}\right)$, where $E$ is a Polish space. Here $\left\{\mathscr{F}_{t}\right\}_{t \geq 0}$ is a filtration and let $\mathscr{F}=\bigvee_{t \geq 0} \mathscr{F}_{t}$. We assume that realization of $X$ is cadlag. By $\mathscr{C}_{b}$ we denote the class of bounded and continuous functions $g: E \rightarrow \mathbb{R}$. We consider now the class $\mathscr{D}(\mathbf{A})$ of all functions $g \in \mathscr{C}_{b}$ such that for some function $g^{*} \in \mathscr{C}_{b}$ the process

$$
M_{g}(t)=g\left(X_{t}\right)-g(x)-\int_{0}^{t} g^{*}\left(X_{s}\right) d s, \quad t \geq 0,
$$

is a $\mathbb{P}_{x}$-martingale. The mapping $g \rightarrow g^{*}$ defines the full generator of the Markov process $X_{t}$ and we denote it by $g^{*}=\mathbf{A} g$. The class $\mathscr{D}(\mathbf{A})$ is called the domain of the full generator $\mathbf{A}$. For $g_{1}, g_{2} \in \mathscr{D}(\mathbf{A})$ denote

$$
\left\langle g_{1}, g_{2}\right\rangle_{A}(x)=\left(\mathbf{A} g_{1} g_{2}\right)(x)-g_{1}(x)\left(\mathbf{A} g_{2}\right)(x)-g_{2}(x)\left(\mathbf{A} g_{1}\right)(x),
$$

provided $g_{1} g_{2} \in D(\mathscr{A})$. The following result was proved in Ethier and Kurtz [(1986), page 175].

Proposition A.1. If $h \in \mathscr{D}(\mathbf{A})$ and $\inf _{x} h(x)>0$, then

$$
M_{t}=\frac{h\left(X_{t}\right)}{h(x)} \exp \left(-\int_{0}^{t} \frac{\mathbf{A} h\left(X_{s}\right)}{h\left(X_{s}\right)} d s\right), \quad t \geq 0,
$$

is a $\mathbb{P}_{x}$-martingale.

Define now a new probability on $(\Omega, \mathscr{T})$ as follows. Let $\mathbb{P}^{t}$ be the restriction of $\mathbb{P}$ to $\mathscr{F}_{t}$ and define on $\mathscr{F}_{t}$ probability $\tilde{\mathbb{P}}_{x}^{t}=M_{t} d \mathbb{P}_{x}^{t}$ for all $t \geq 0$. Since $M_{t}$ is a martingale, the family of probabilities $\left\{\tilde{\mathbb{P}}_{x}^{t}, t \geq 0\right\}$ is consistent. From the Daniel-Kolmogorov theorem, we get that there exists the unique probability $\tilde{\mathbb{P}}_{x}$ such that for each $t \geq 0$ the restriction of $\tilde{\mathbb{P}}_{x}$ to $\mathscr{T}_{t}$ is $\tilde{\mathbb{P}}_{x}^{t}$, $x \in \mathbb{R}$. Moreover, from Kunita (1976), the process $X(t)$ is a Markov process on $\left(\Omega, \mathscr{F},\left\{\mathscr{F}_{t}\right\}_{t \geq 0},\left\{\tilde{\mathbb{P}}_{x}\right\}_{x \in E}\right)$.

Proposition A.2. Let $h \in \mathscr{D}(\mathbf{A})$ be such that $g h \in \mathscr{D}(\mathbf{A})$ for all $g \in \mathscr{D}(\mathbf{A})$ and $\inf _{x} h(x)>0$. Then the process $X$ on $\left(\Omega, \mathscr{F},\left\{\mathscr{F}_{t}\right\}_{t \geq 0},\left\{\tilde{\mathbb{P}}_{x}\right\}_{x \in E}\right)$ is a Markov family with generator

$$
\tilde{\mathbf{A}} g(x)=\mathbf{A} g(x)+\frac{\langle h, g\rangle_{A}(x)}{h(x)}
$$

and domain $\mathscr{D}(\tilde{\mathbf{A}})=\mathscr{D}(\mathbf{A})$.

Acknowledgment. We thank the referee for remarks and, in particular, for an observation that resulted in a shorter proof of Theorem 1.1. 


\section{REFERENCES}

ANICK, D., MitrA, D. and SondHI, M. M. (1982). Stochastic theory of a data-handling system with multiple sources. Bell System Technical Journal 61 1871-1894.

Asmussen, S. (1994). Busy period analysis, rare events and transient behavior in fluid flow models. J. Appl. Math. Stochastic Anal. 7 269-299.

Asmussen, S. and Rubinstein, R. Y. (1995). Steady state rare events simulation in queueing models and its complexity properties. In Advances in Queueing (J. Dhashalow, ed.) 429-462. CRC Press, Boca Raton, FL.

Bensaou, B., Guibert, J., Roberts, J. W. and Simonian, A. (1994). Performance of an ATM multiplexer queue in the fluid approximation using the Benes approach. Ann. Oper. Res. 49 137-160.

Davis, M. H. A. (1984). Piecewise-deterministic Markov processes: a general class of non-diffusion stochastic models (with discussion). J. Roy. Statist. Soc. Ser. B 46 353-388.

Davis, M. H. A. (1993). Markov Models and Optimization. Chapman and Hall, London.

DĘBICKI, K. and Rolski, T. (1995). A Gaussian fluid model. Queueing Systems Theory Appl. 20 433-452.

EthieR, H. J. and KURTZ, T. G. (1986). Markov Processes, Characterization and Convergence. Wiley, New York.

EthieR, H. J. and KURTz, T. G. (1993). Fleming-Viot processes in population genetics. SIAM J. Control Optim. 31 345-386.

Fukushima, M. and Stroock, D. (1986). Reversibility of solutions to martingale problems. Adv. Math. Suppl. Stud. 9 107-123.

GUIBERT, J. (1994). Overflow probability upper bound in fluid queues with general on-off sources. J. Appl. Probab. 31 1134-1140.

Guibert, J. and Simonian, A. (1995). Large deviations approximation for fluid queues fed by a large number of on/off sources. IEEE Journal on Selected Areas in Communications 13 1017-1027.

HeAth, D., Resnick, S. and SAmorodnitsky, G. (1996). Heavy tails and long range dependence in on/off processes and associated fluid models. Preprint, Cornell Univ., Ithaca, NY.

Jelenkovič, P. R. and LAZAR, A. A. (1996). Multiplexing on-off sources with subexponential on periods. Preprint, Columbia Univ., New York.

Kingman, J. F. C. (1961). A convexity property of positive matrices. Quart. J. Math. Oxford Ser. (2) 12 283-284.

Kopociński, B. (1967). Breakdown processes of systems in series. Appl. Math. 9 149-160.

KopocińsKi, B. (1973). Zarys Teorii Odnowy i Niezawodności. PWN, Warszawa.

KULKARNI, V. G. (1994). Effective bandwidth for Markovian regenerative sources. Technical Report UNC/OR/TR94-13, Dept. Operations Research, Univ. North Carolina, Chapel Hill.

Kulkarni, V. G. (1995). Fluid models for single buffer systems. In Advances in Queueing (J. Dhashalow, ed.). CRC Press, Boca Raton, FL.

KULKARNI, V. and Rolski, T. (1994). Fluid model driven by an Ornstein-Uhlenbeck process. Probab. Engrg. Inform. Sci. 8 403-417.

Kunita, H. (1976). Absolute continuity of Markov processes. Seminaire de Probabilites X. Lecture Notes in Math. 511 44-75. Springer, Berlin.

Neuts, M. F. (1981). Matrix-Geometric Solutions in Stochastic Models; An Algorithmic Approach. Johns Hopkins Univ. Press.

PalmowsKi, Z. (1996). A note on the exponential change of measure. Manuscript, Mathematical Institute, Wrocław Univ., 1996.

Palmowski, Z. and Rolski, T. (1996). A note on martingale inequalities for fluid models. Statist. Probab. Lett. 31 13-21.

Shwartz, A. and Weiss, A. (1995). Large Deviations for Performance Analysis. Queues, Communications, and Computing. Chapman and Hall, London.

STERN, T. E. and ElWALID, A. I. (1991). Analysis of separable Markov-modulated rate models for information handling systems. Adv. in Appl. Probab. 23 105-139. 
SzczotKa, W. (1980). Central limit theorem in $D[0, \infty)$ for breakdown processes. Probab. Math. Statist. 1 49-57.

SzEkLI, R. (1995). Stochastic Ordering and Dependence in Applied Probability. Springer, New York.

WHITT, W. (1993). Tail probabilities with statistical multiplexing and effective bandwidths in multi-class queues. Telecommunication Systems 2 71-107.

MATHEMATICAL Institute

UNIVERSITY OF WROCŁAW

PL. GRUNWALDZKI 2/4

50-384 WROCŁAW

POLAND

E-MAIL: zpalma@math.uni.wroc.pl rolski@math.uni.wroc.pl 